\title{
System identification of DM4 glomerulus in the Drosophila antennal lobe using stationary and non-stationary odor stimuli
}

\author{
Anmo J Kim, Aurel A Lazar*, Yevgeniy Slutskiy \\ From Nineteenth Annual Computational Neuroscience Meeting: CNS*2010 \\ San Antonio, TX, USA. 24-30 July 2010
}

The lack of precise stimulus delivery and measurement systems has fundamentally limited the progress of system identification in olfaction. In [1], we used a novel in vivo experimental setup with precise and reproducible delivery of airborne stimuli to apply system identification methods to Olfactory Sensory Neurons (OSNs) in Drosophila. Here we continue our investigation of the olfactory coding problem by applying the same time-varying odor stimuli and recording in vivo the response of Projection Neurons (PNs) postsynaptic to OSNs in [1].

\section{Results}

First, we applied white noise odor waveforms and recorded the response of PNs innervating the DM4 glomerulus. Using the obtained odorant-response input/output pairs we constructed a one-dimensional Linear-Nonlinear (1D LN) cascade model that can faithfully predict the measured average spike rates. However, both the linear kernel and the nonlinearity of the model change with the mean concentration and the variance of a white noise stimulus. This dependence of model parameters on test stimuli suggested us to consider higher dimensional models.

Next, we used the spike-triggered covariance (STC) method to build a two-dimensional LN (2D LN) cascade model for the OSN spike train-PN spike train input/output pair. The first principal eigenvector of the STC matrix is monophasic and tuned to the slow amplitude component of the input. At the same time, the second principal eigenvector is biphasic and tuned to the variance component of the input. Although the model closely predicts PN output for white noise waveforms, it fails to predict PN output for non-stationary odor

Department of Electrical Engineering, Columbia University, New York, NY 10027, USA waveforms. By analyzing the trajectory of white noise stimuli, we determined that the predictive power of the 2D LN model is adversely affected by the limitations imposed on the set of input odor waveforms.

Finally, we estimated the non-linear block of the 2D LN model using non-stationary triangle odor waveforms with bigger trajectories and propose a model that can predict PN output to a larger set of stimuli. Combining the resulting model for the DM4 glomerulus with the OSN model in [1], we demonstrate that PNs most strongly encode information about both the first and the second derivative of odor concentration.

\section{Conclusions}

We build a 2D LN model of the DM4 glomerulus in Drosophila antennal lobe using both white noise and non-stationary odor waveforms. The resulting model faithfully predicts the output of PNs and suggests a strong temporal processing of odor information in the early olfactory system of the fruit fly.

\section{Acknowledgements \\ The work presented here was supported by $\mathrm{NIH}$ under grant number R01DC008701-01 and was conducted in the Axel laboratory at Columbia University. The authors would like to thank Dr. Richard Axel for insightful discussions and for his outstanding support.}

Published: 20 July 2010

\section{Reference \\ 1. Kim J.Anmo, Lazar A.Aurel, Slutskiy Yevgeniy: 2D Encoding of Concentration and Concentration Gradient in Drosophila ORNs,. Computational and Systems Neuroscience Meeting 2010, COSYNE 2010, Salt Lake City, UT. \\ doi:10.1186/1471-2202-11-S1-P174 \\ Cite this article as: Kim et al:: System identification of DM4 glomerulus in the Drosophila antennal lobe using stationary and non-stationary odor stimuli. BMC Neuroscience 2010 11(Suppl 1):P174.}

